\title{
L'ÉCOLE DE GENÈVE
}

\section{Vue de la Société de linguistique de Paris}

\section{Pierre-Yves Testenoire}

Université Sorbonne Nouvelle Paris 3

Laboratoire d'Histoire des théories linguistiques (UMR 7597 du CNRS)

\section{Résumé}

Cet article entend contribuer à la réflexion sur les caractéristiques de l'école linguistique de Genève, par le biais d'un regard extérieur : celui de la Société de linguistique de Paris. L'école dite " de Genève » et la Société de linguistique de Paris ont évidemment partie liée. Les points de contact et les passerelles entre les deux ne manquent pas. Elles partagent également de nombreux postulats et un héritage commun, quoique plurivoque : l'enseignement saussurien. Reconnait-on pour autant, au sein de la Société de linguistique de Paris, une spécificité ou une cohérence propre à l'approche linguistique développée à Genève ? Y a-t-il entre les élèves directs et indirects de Saussure à Paris et ses disciples genevois le constat de différences, de désaccords ? Pour répondre à ces questions, l'article se fonde sur le dépouillement des Bulletins de la Société de linguistique de Paris, et tout particulièrement de sa section de comptes-rendus, entre 1900 et 1940.

\section{Mots-clés}

Bally, école de Genève, école linguistique, linguistique française, Meillet, Sechehaye

\section{Abstract}

The aim of this article is to contribute an outside perspective, that of the Sociéte de linguistique de Paris, to the study of the characteristics of the Geneva school of linguistics, The School of Geneva and the Société de linguistique de Paris are linked, with numerous areas of contact and connections between them. They also share the postulates and common heritage of Saussurian teaching. Does the Société linguistique de Paris acknowledge the specificity of the linguistic approach developed in Geneva? Is difference and disagreement accepted between direct and indirect students of Saussure in Paris and his disciples in Geneva? This article is based on the analysis of the Bulletins de la Société de linguistique de Paris, and particularly on the section on reports, between 1900 and 1940 .

\section{Keywords}

Bally, French linguistics, Geneva School, Meillet, School of linguistics, Sechehaye 
L'objet de cet article est d'observer comment le développement de la linguistique à Genève a été reçu et évalué par la Société de linguistique de Paris entre 1900 et 1940. Entre ces deux dates, le syntagme nominal " école linguistique de Genève » est passé de l'indéfini au défini, transformant une formule convenue lancée par Michel Bréal lors d'un banquet en $1894^{1}$ en l'appellation validée par les linguistes pour désigner une communauté de chercheurs et une communauté de pensée, reconnues comme telles et dotées, en définitive, d'une société savante et d'une revue. La période considérée est donc celle de l'émergence de l'école genevoise jusqu'à sa première institutionnalisation. Les bornes chronologiques de cette enquête correspondent à des moments charnières pour l'histoire sociale de la linguistique à la fois en France et en Suisse. Si la première décennie du siècle voit à Genève ce moment séminal des dernières années de l'enseignement saussurien, elle coïncide également, à Paris, avec l'accession d'Antoine Meillet à la chaire de Grammaire comparée du Collège de France et au poste de secrétaire adjoint de la Société de linguistique de Paris (1906). Cette décennie inaugure ainsi ce que l'historiographie contemporaine a coutume d'appeler « le règne de Meillet sur la Société » jusque dans les années 1930 (Lamberterie 2005, p. 41). L'année 1940, enfin, où nous nous arrêterons, voit simultanément la suspension des activités de la Société de linguistique de Paris à cause de l'invasion allemande et, en Suisse, la création de la Société Genevoise de Linguistique et de sa revue, les Cahiers Ferdinand de Saussure.

\section{La Société ET/OU L’ÉCOLE}

L'étude de la réception de la production linguistique genevoise en France ne peut faire l'économie des catégories d' " école de Paris », d' " école française de linguistique » ou d'« école linguistique française »- les trois syntagmes se rencontrent - utilisées pour désigner précisément le courant de la linguistique historique, à prétention sociologique, qui se développe en France dans le premier tiers du $\mathrm{xx}^{\mathrm{e}}$ siècle. L'expression émerge dans la foulée de l'accession de Meillet au Collège de France. La paternité semble revenir aux linguistes français euxmêmes - Meillet 1906, Grammont 1912, Gauthiot 1914, Ernout 1922, Cahen 1925, Meillet 1928, Boyer 1936... La catégorie de l'« école de Paris » est

1 L'anecdote est rapportée par Bally en 1908: «Au Congrès des Orientalistes qui s'est tenu à Genève en 1894 et où vous [Saussure] avez fait une importante communication, $\mathrm{M}$. Michel Bréal, au cours d'un banquet, a salué en vous le fondateur d'une école genevoise de linguistique. Je me souviens qu'alors votre modestie accueillit ces paroles avec un scepticisme souriant. » (Meillet Bally [1908] 2006, p. 185). Comme le fait remarquer Joseph, " the scepticism was undoubtedly there, not unmixed with some embarrassment, since those present from his own Université knew that, if a "school " could be said to be forming, it had almost no students » (2012, p. 407). Les étudiants de Saussure à Genève dans les premières années de son enseignement se comptent effectivement sur les doigts d'une seule main. 
reprise par ceux qui cherchent à s'en démarquer - Sechehaye 1927 - ou à s'en revendiquer de l'extérieur - Sommerfelt 1924, 1932. Le succès de l'expression ne garantit pas pour autant sa consistance. Dans les descriptions contemporaines de cette école, les deux paramètres généralement tenus pour définitoires d'une école de pensée ou d'une école de recherche - la convergence sociale et la convergence théorique $^{2}$ - se signalent par leur labilité. Les principes théoriques communs sont passés sous silence ou réduits à des généralités, divergentes d'un auteur à l'autre : ce qui unit les membres de l'école est " l'influence de leur maître [Saussure]" pour Gauthiot tandis que pour Sommerfelt c'est leur " point de vue sociologique ». Quant aux contours sociaux de l'école, ils ne sont pas moins flous. Ainsi que l'a montré Savatovsky (2004), la liste des membres varie en fonction des acteurs de l'école eux-mêmes, chacun retenant des critères d'inclusion selon des stratégies mémorielles ou de légitimation propres. C'est en raison de cette indétermination conceptuelle et sociale que nous n'utiliserons pas la catégorie d' 'école linguistique de Paris ». Alors même que la validité de la notion d'école linguistique en général et d'école de Genève en particulier se doit d'être interrogée, postuler une " école de Paris ", dont les contours paraissent encore plus flous, reviendrait à redoubler le problème sans l'éclaircir. Le prisme retenu pour l'analyse de la réception française de la linguistique genevoise sera celui de la Société de linguistique de Paris. Une société savante présente sur l'école un avantage empirique : celui d'être observable de manière moins médiatisée. La SLP a, en effet, des membres, des statuts, un bureau, des séances de travail avec des comptes-rendus et des organes de diffusion : les Mémoires, les Bulletins, une collection « Linguistique »... Par rapport aux contours nécessairement flous d'une école, produits par les représentations de ses partisans comme de ses adversaires, le cadre institutionnel d'une société savante présente donc des frontières nettes aussi bien en synchronie - qui en est, qui n'en est pas à un moment $\mathrm{T}$ - qu'en diachronie - depuis sa fondation jusqu'à son éventuelle dissolution.

Substituer à la problématique de l'école celle de la société savante revient en apparence à déplacer un questionnement épistémologique sur le terrain social et institutionnel. L'appréhension d'un ensemble de travaux dont on perçoit une unité du point de vue de la société savante qui les réunit contourne effectivement en partie le problème de la ligne doctrinale que pose l'appellation d'école. Les membres de la SLP partagent des "règles » : ce sont celles des statuts de la société. Un seul article dans les statuts de 1876, en vigueur pour la période qui nous intéresse, relève de l'orientation scientifique. C'est l'article premier qui stipule que « la Société de Linguistique a pour objet l'étude des langues et l'histoire du langage » et que « tout autre sujet d'études est rigoureusement interdit». Dans ce

2 Cf. par ex. Amsterdamska 1987, p. 4-31, Servos 1993 et Waquet 2008, p. 271-281. 
cadre très général, rien ne détermine un ensemble de postulats que les membres de la Société devraient partager. Dès lors, les travaux de la SLP ne présentent pas d'unité théorique et, de fait, dans l'entre-deux-guerres, cohabitent en son sein des savants aux perspectives aussi différentes et aux désaccords aussi profonds que Grammont et Martinet, Pichon ou Guillaume. Si les statuts ne déterminent pas une ligne doctrinale a priori, il est possible de cartographier pour chaque membre des degrés d'intégration au sein de la Société à partir de critères que fournit l'institution : assiduité aux séances, nombre de publications dans les organes de la société, travaux cités ou recensés dans les Bulletins... La centralité d'un Meillet, assurant certaines années la totalité de la section des comptes-rendus des $B S L$ - allant jusqu'à rendre compte de ses propres ouvrages - ou la marginalité d'un Pichon qui, bien que membre assidu pendant près de dix ans, n'y publie jamais renseignent aussi sur l'orientation scientifique de la SLP. Si tous les secrétaires depuis Bréal ont constamment réaffirmé que la SLP n'a « sur aucun point de doctrine arrêtée » (Vendryes 1955, p. 21), la question de l'identification de la société à une école de recherche ne reçoit pas sur la même période une réponse constante. Devenue, à la fin des années 1870, l'un des ancrages institutionnels du petit groupe de comparatistes de l'enseignement supérieur français, principalement réunis à l'EPHE, la SLP voit jusqu'à la Première Guerre mondiale ses travaux identifiés aux activités d'une école de Paris : " Comme le dira M. M. Pavlovic (Institut de la langue serbo-croate) dans son discours pour la commémoration de la SLP : quand on parle de la linguistique qui se fait en France, on ne parle pas d'école de Paris mais tout uniment de la SLP » (Bergounioux 2005, p. 367). En 1914, Gauthiot écrit naturellement que l'une des caractéristiques des membres de l'école linguistique de Paris est « leur groupement dans la Société de linguiste de Paris » (1914, p. 54). Après-guerre, la SLP, à mesure de son internationalisation et du développement par le monde d'autres sociétés linguistiques, récuse pour elle-même le terme d'école. C'est ainsi que Meillet plaide en 1926 pour l'abandon du syntagme « école française » qu'il utilisait jusque-làa :

En vérité il sera bon d'éviter les termes de : «école allemande » et « école française "; la science souffre trop des amours-propres personnels, qui sont irrémédiables, pour qu'on n'évite pas de souligner des oppositions nationales qu'on peut éviter et qui, en l'espèce, ne sont pas graves : en linguistique, les hommes ont toujours eu assez d'esprit - et de bon sens - pour ne pas faire état des différences de nationalité et pour n'envisager que les faits. (Meillet 1926-27, p. 65).

À la veille du premier congrès international de linguistes, le comparatisme français a pour la science allemande la commisération des vainqueurs. Ce sont à peu près les mêmes arguments qu'utilise Vendryes en conclusion de son article de 1955 
sur la « première société linguistique » pour récuser toute identification de la SLP à une école linguistique :

L'atmosphère dans laquelle la Société a vécu a été faite de justice et de politesse dans les relations entre confrères. Il n'est est pas de plus favorable aux intérêts du travail scientifique. Grâce à elle, ce travail s'accomplit aisément, sans effort et sans heurt, par une bonne entente entre gens de bonne foi, qui mettent au dessus de tout leur commun amour de la science. La Société de linguistique de Paris, qui est sans doute aujourd'hui la plus ancienne du monde, ne se réclame d'aucune doctrine rigide et exclusive. Elle ne représente même pas une école linguistique, à moins qu'on n'entende sous ce nom un simple accord entre linguistes pour la recherche de la vérité. (Vendryes 1955, p. 21)

La présentation ne rend compte ni des conflits théoriques qui traversent la SLP - avec le développement de la phonologie par exemple (cf. Chevalier 1997) -, ni de l'hégémonie au sein de la société d'une discipline (la grammaire comparée) et d'un courant philosophique (l'empirisme). L'irénisme dont fait preuve ici Vendryes traduit surtout la conscience que la Société de linguistique de Paris a de sa primauté - elle est « la plus ancienne du monde »- et de sa centralité. Quelques années plus tard, Benveniste porte une appréciation plus nuancée sur la SLP sous le magistère de Meillet :

Meillet avait fait des séances de la Société comme un prolongement de son enseignement. Il y apportait selon l'occasion des étymologies, des remarques de détail ou des exposés d'ensemble, et c'est là que, à leur tour, ses élèves présentaient leurs propres recherches, en général dans la ligne de ses travaux. Les séances devenaient, dans cette unité d'inspiration, comme une seconde école où maints jeunes comparatistes, et d'autres moins jeunes, achevaient de se former. (Benveniste 1971, p. 31)

Mais si Benveniste reconnaît que les travaux de la SLP ont pu correspondre à une école linguistique dont Antoine Meillet aurait été le chef de file, c'est pour mieux souligner que le temps de cette homogénéisation doctrinale est révolu :

Chacun de nous a des préférences doctrinales, son orientation personnelle. Mais il ne peut y assujettir la Société. Le rôle d'une Société n'est pas d'enseigner une vérité, mais d'encourager la libre recherche [...]. Cette ligne de conduite qui a été celle de nos prédécesseurs, est plus que jamais nécessaire [...]. (Ibid., p. 34)

Les reconstitutions de Vendryes et de Benveniste, qui témoignent du discrédit attaché au concept d' " école scientifique » perçu comme oxymorique, mettent l'accent sur l'ouverture de la SLP en phase avec le processus d'institutionnalisation de la science linguistique à l'échelle mondiale (Chevalier 2000). Pour autant, l'ambition fédérative de la SLP qui s'affirme dans l'entre-deux-guerres ne se départ pas d'une certaine ambivalence : si elle prétend transcender les écoles, et l'école française en particulier, ses publications identifient bien la linguistique qui se développe ailleurs en Europe comme extérieure. 


\section{DeUX SOCIÉTÉS SCEURS ?}

Jusqu'en 1940, la linguistique à Genève ne bénéficie pas d'ancrages institutionnels comparables à ceux de Paris. En dehors des rares postes que fournit l'Université4, la linguistique ne s'appuie à Genève sur aucune société, revue ou collection spécifiques. Mettre en regard pour la période considérée une « école de Paris » et une « école de Genève » reviendrait à établir une forme d'équivalence là où la relation, d'un point de vue institutionnel, est profondément asymétrique. Les deux expressions, par la polarité géographique qu'elles induisent, établissent entre les deux un rapport d'extériorité. Or l'école de Genève et la Société de linguistique de Paris sont dans un rapport d'inclusion réciproque.

Leur étroite imbrication est d'abord observable dans l'itinéraire de leurs membres. Les principaux linguistes que l'on rattache à l'école de Genève sont membres de la SLP : Bally en est membre depuis 1900, où il est présenté par Saussure et Meillet. Sechehaye est présenté plus tardivement, en 1917, soit un an après la parution du Cours de linguistique générale. André Burger devient membre en 1922, Serge Karcevski en 1925. Les parcours de ces savants attestent une circulation incessante entre la Suisse et la France, brouillant une hypothétique ligne de partage entre la linguistique genevoise et la SLP. Bally fait ainsi en 1903-1904 un séjour d'étude à l'EPHE lors duquel il participe assidûment aux séances de la SLP. Paul Regard, auditeur des cours de linguistique générale de Saussure, poursuit ensuite ses études à l'EPHE sous la direction d'Antoine Meillet. André Burger, qui effectue toute sa carrière à Neuchâtel puis à Genève, écrit qu'il doit "l'essentiel de [sa] formation linguistique à [son] maître, M. A. Meillet » (Segre 1985, p. 3). Quant à Jules Ronjat, privat-docent à Genève de 1915 à 1925 et qui, à ce titre, aide Bally et Sechehaye dans l'exploration des manuscrits saussuriens, il inscrit surtout ses travaux en romanistique et sur le langage enfantin dans la lignée de ceux de Maurice Grammont. Les publications témoignent également des liens inextricables entre la linguistique qui se fait à Genève et la SLP : les Mélanges de linguistique offerts à Ferdinand de Saussure forment par exemple le deuxième titre de la collection "linguistique » de la SLP, l'Essai sur la structure logique de la phrase de Sechehaye, le vingtième. C'est donc bien la circulation qui prévaut entre les cercles linguistiques de l'entre-deux-guerres, circulation incarnée par la figure de Karcevski, ancien élève de Saussure, qui est à la fois membre de la SLP, membre fondateur du Cercle de Prague, et l'un des initiateurs de la Société Genevoise de Linguistique.

4 Pour un état des lieux institutionnel de la linguistique et de la philologie à l'Université de Genève entre 1872 et 1945, on consultera Fryba-Reber 2013, p. 255-300. 
La Société de linguistique de Paris, réalisant véritablement sa vocation internationaliste dans l'entre-deux-guerres, est donc bel et bien constitutive de l'école linguistique genevoise. Elle s'en disjoint néanmoins lorsqu'est fondée, en 1940, la Société Genevoise de Linguistique. L'initiative en revient à deux professeurs de la génération qui suit celle de Bally et de Sechehaye : Henri Frei et Serge Karcevski. Ce dernier organise en mai et juin 1940 plusieurs réunions informelles où des exposés linguistiques sont discutés. Le 21 décembre 1940 a lieu l'Assemblée constituante de la Société Genevoise de Linguistique. Il ne paraît pas anodin que l'école genevoise s'institutionnalise l'année même où la SLP suspend ses activités. Dans les textes qui accompagnent la création de la nouvelle société, les exemples revendiqués par les genevois sont le Cercle de Prague et le Cercle de Copenhague, la SLP n'étant jamais mentionnée. C'est le cas par exemple de l'état des lieux que dresse Sechehaye de «l'influence saussurienne », où Paris n'occupe aucune place :

Grâce à Ferdinand de Saussure le nom de Genève occupe une place en vue dans l'histoire de la science linguistique du début de ce siècle. Son ouvrage posthume, le Cours de linguistique générale, extrait des cahiers de ses élèves, a exercé une influence décisive, qui s'affirme de plus en plus, sur la pensée de tous ceux que préoccupent les problèmes de la langue. En outre, quelques-uns de ses disciples genevois, fortement marqués de son empreinte, mais créateurs à leur tour selon leurs tempéraments respectifs, ont continué son action et l'on a pu parler d'une école genevoise de linguistique.

Mais l'influence saussurienne s'est affirmée ailleurs en provoquant de fécondes initiatives. Nous pensons au Cercle de Prague, né en 1926, et à ses Travaux, rédigés par une belle phalange de collaborateurs grâce auxquels une discipline nouvelle, et combien intéressante, la "Phonologie » a conquis sa place dans la linguistique moderne. Nous pensons aussi au petit groupe des linguistes de Copenhague qui ont créé une nouvelle revue, les Acta linguistica, sur le programme de la « linguistique structurale » et qui, aujourd'hui encore, poursuivent leur effort malgré toutes les difficultés du moment.

Ces deux mouvements n'étaient-ils pas, pour nous autres Genevois, des exemples ? C'est là ce qu'a pensé M. Henri Frei, qui, revenu récemment d'Extrême-Orient, après un long séjour consacré à l'étude et à l'enseignement, vient de succéder à son maître $\mathrm{M}$. Charles Bally dans la chaire de Grammaire comparée et de sanscrit.

Sous son impulsion les linguistes genevois ont été groupés, des rencontres scientifiques ont été organisées et, en décembre dernier, la Société Genevoise de Linguistique a été fondée. (Sechehaye 1941, p. 175-176)

Sechehaye attribue, en outre, l'initiative de fonder une société au seul linguiste titulaire de l'Université de Genève qui n'est pas membre de la SLP : Henri Frei. Son absence au sein de la SLP trouve vraisemblablement son origine dans la recension très sévère que Meillet a faite de La Grammaire des fautes, Henri Frei s'étant depuis lors toujours abstenu de le citer (Amacker et Godel 1980, p. 122).

À la reprise des activités de la SLP en 1945, les Bulletins de la Société de linguistique de Paris (désormais BSL) consacrent leur premier compte-rendu aux premières livraisons des Cahiers de Ferdinand de Saussure. Sous la plume de 
Marcel Cohen, la SLP souhaite alors « la plus cordiale bienvenue à la nouvelle société sœur, et à son périodique » $(1945$, p. 1). C'est donc la perception de cette sororité, un peu siamoise, nous l'avons vu, qui nous intéresse en amont de l'autonomisation de 1940 .

\section{LA PRODUCTION LINGUISTIQUE GENEVOISE JUSQU'EN 1940}

Pour analyser la manière dont la Société de linguistique de Paris conçoit l'émergence de cette école de pensée qu'elle héberge en partie en son sein, nous nous fondons sur le dépouillement de la section des comptes-rendus des BSL. Le corpus des comptes-rendus et des ouvrages analysés est récapitulé ci-dessous ( $c f$. fig. 1). Ce tableau est une présentation chronologique et synoptique de la production linguistique à Genève sur la période considérée. Il commence en 1908, date de la création par Antoine Meillet de la section « comptes-rendus critiques » des $B S L$; il est organisé en deux colonnes selon les deux mouvements qui traversent toute école de pensée : celui, excentrique, de la divergence, inhérent à la pluralité des membres qui la composent, et celui, concentrique, de la convergence théorique. La troisième colonne contient, en regard de chaque publication, le compte-rendu paru dans les $B S L$ avec le nom de l'auteur, le tome et la pagination.

Dans la première colonne du tableau, figurent les principales publications individuelles de linguistes genevois qui traduisent autant d'orientations scientifiques potentiellement diverses. La liste ne recense donc pas tous les ouvrages de linguistique parus à Genève entre 1908 et 1940, mais seulement les ouvrages ou les numéros de revue réunissant plusieurs contributions de linguistes genevois recensés dans les $B S L$. On y trouve les livres des linguistes exerçant à Genève, Charles Bally et Albert Sechehaye, mais aussi les publications de thèses d'élèves moins connus de Saussure - Léopold Gautier et Paul Regard - et d'élèves de Bally - Marguerite Lips, Henri Frei et Hans Adank. Le livre de Karcevski sur le Système du verbe russe a été pris en compte, bien que son lieu de publication soit Prague, parce que son auteur exerce alors déjà comme chargé de cours à l'Université de Genève et parce que le compte-rendu insiste sur sa filiation saussurienne. Le seul linguiste intégré à cette liste qui n'ait pas de contact géographique avec Genève est le Hollandais Cornelis De Boer. Son intégration à l' " école genevoise » se fait sur une base théorique, revendiquée par l'auteur dans ses ouvrages - son Introduction à l'étude de la syntaxe du français est dédiée à Albert Sechehaye - et reconnue dans les comptes-rendus des $B S L$.

Par rapport à ces productions individuelles, la deuxième colonne réunit donc des publications, le plus souvent collectives, qui témoignent d'un mouvement unitaire ou concentrique. Les publications ou activités réunies dans cette colonne 
sont hétérogènes et peuvent être réparties en quatre catégories, quatre manières complémentaires de faire école.

La première passe par la constitution posthume de la figure du chef d'école à partir d'un corpus de textes stabilisé. Relève de cette catégorie tout le travail éditorial autour des textes de Saussure : la parution du Cours de linguistique générale en 1916, ses rééditions en 1922 et 1931 et la constitution du Recueil des publications scientifiques de Saussure en 1922. Significativement, l'origine commune que se choisissent les promoteurs de l'école se situe dans un évènement : l'enseignement de Saussure à Genève. L'assignation à une parole plutôt qu'à un écrit, à un lieu excentré - Genève - et à un petit nombre de privilégiés active le clivage de l'ésotérique et de l'exotérique, cimentant la cohésion du groupe.

La deuxième catégorie d'initiatives concentriques consiste à réunir des chercheurs partageant une communauté de pensée sans pour autant solliciter dans leur contribution des convergences théoriques. Trois initiatives relèvent de cette catégorie : les Mélanges de linguistique offerts à Ferdinand de Saussure en 1908, à l'occasion d'une petite fête qui réunit ses élèves directs et indirects, l'organisation du $2^{\mathrm{e}}$ Congrès International de Linguistes à Genève en 1931 et les Mélanges de linguistique offerts à Charles Bally en 1939 pour son départ à la retraite. Chacune de ces initiatives est l'occasion de rappeler à la communauté savante réunie l'identité d'une « école genevoise » (Meillet Bally [1908] 2006 ; p. 185, Bally 1933, p. 2930 ; Sechehaye [1939] 2001, p. 461) et pour les membres revendiqués de cette école de se compter. Ce type d'initiatives permet au groupe d'affirmer à la fois son identité et son ouverture vis-à-vis du reste de la communauté scientifique, participant pleinement du système de double légitimation analysé par Olga Amsterdamska (1987, p. 19-27) dans la constitution des écoles scientifiques.

Une troisième catégorie d'initiatives peut être distinguée sur le critère du degré supplémentaire d'intégration demandé aux participants ; elle regroupe les publications collectives qui reposent sur une convergence théorique. Pour la période considérée, deux publications relèvent de cette catégorie. La première est la contribution commune élaborée par Bally et Sechehaye pour la quatrième question soumise

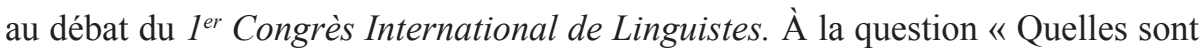
les méthodes les mieux appropriées à un exposé complet et pratique de la grammaire d'une langue quelconque ? » les deux linguistes répondent par un point de vue unitaire, en exposant « les doctrines de l'école genevoise de linguistique [qui] pourraient contribuer à sa solution » (Bally et Sechehaye 1930, p. 36). On sait, par le témoignage de Sechehaye, que cette position commune est le résultat d'une âpre négociation entre les deux linguistes (Fryba-Reber 2001, p. 443-452). Cette contribution est exceptionnelle dans les Actes du congrès, par son caractère collectif d'abord - elle est la seule avec celle du Cercle Linguistique de Prague à compter 
plusieurs signataires -, par son format ensuite : elle fait dix-huit pages quand les autres réponses n'excèdent pas cinq pages. Son rôle paraît décisif dans la perception d'une école genevoise : ce dont témoignent la décision d'organiser le congrès suivant à Genève et la diffusion de l'expression « école genevoise de linguistique » qui se répand à partir de la fin des années 1920. La seconde contribution collective est l'article " Pour l'arbitraire du signe » de 1940 signé par Sechehaye, Bally et Frei en réponse aux articles de Benveniste (1939) et de Pichon (1940) parus dans Acta linguistica. Comme pour la contribution au congrès de La Haye, la démarche unitaire, le titre et le ton offensifs donnent à ce texte le caractère d'un manifeste d'école.

Enfin, il convient d'isoler une quatrième catégorie qui ne se distingue de la précédente que parce qu'elle émane d'une initiative individuelle. Ne rentrent dans cette catégorie que certains discours ou publications d'Albert Sechehaye, au premier rang desquels l'article "L'école genevoise de linguistique générale » paru en 1927. À partir d'un exposé des travaux de Saussure, de Bally et des siens, Sechehaye opère une synthèse doctrinale. Dans cette entreprise, l'hétérogénéité des travaux des trois linguistes est analysée en termes de répartition des tâches, gage de cohérence de l'école. Sechehaye insiste tout particulièrement sur la complémentarité de son approche et de celle de Bally : chacun étudie les faits linguistiques «par l'autre bout de la lunette », illustrant ainsi « les deux pôles du langage » que sont la vie et la logique (1927, p. 235). En définitive, l'école genevoise se caractérise, selon lui, par « l'union de deux tendances, en apparence contradictoires » : « celle qui considère la linguistique comme une science aux principes abstraits» et « celle qui vise à mettre cette science au service des fins les plus pratiques », notamment pédagogiques (Ibid., p. 239-240). Avec cet article comme avec la contribution collective au congrès de La Haye dont il est l'initiateur, Sechehaye fait figure de véritable promoteur de l'école de Genève.

\begin{tabular}{l|l|l|l} 
& Mouvement excentrique & Mouvement concentrique & $\begin{array}{l}\text { Comptes-rendus } \\
\text { BSL }\end{array}$ \\
\hline 1908 & $\begin{array}{l}\text { Sechehaye, Programme et méthode de la } \\
\text { linguistique théorique. Psychologie du } \\
\text { langage }\end{array}$ & Mélanges Saussure & Havet T. 16, p. 21-54 \\
\hline 1909 & $\begin{array}{l}\text { Bally, Traité de stylistique française } \\
\text { Sechehaye, Éléments de grammaire } \\
\text { historique du français }\end{array}$ & & Meillet T. 16, p. 118-122 \\
\hline 1911 & Gautier, La langue de Xénophon & & Meillet T. 17, p. 59-60 \\
\hline 1913 & Bally, Le langage et la vie & Cours de linguistique générale & Meillet T. 20, p. 32-36 \\
\hline 1916 & & &
\end{tabular}




\begin{tabular}{|c|c|c|c|}
\hline & Mouvement excentrique & Mouvement concentrique & $\begin{array}{l}\text { Comptes-rendus } \\
\text { BSL }\end{array}$ \\
\hline 1919 & $\begin{array}{l}\text { Regard, La phrase nominale dans } \\
\text { la langue du nouveau testament et } \\
\text { Contribution à l'étude des prépositions } \\
\text { dans la langue du Nouveau Testament }\end{array}$ & & Meillet T. 21, p. 212-215 \\
\hline 1921 & $\begin{array}{l}\text { Bally, Traité de stylistique française, } 2^{\mathrm{e}} \\
\text { édition } \\
\text { Journal de Psychologie. Psychologie } \\
\text { du langage (articles de Bally, Lips et } \\
\text { Sechehaye) }\end{array}$ & & $\begin{array}{l}\text { Meillet T. } 22 / 2 \text {, p. } 232 \\
\text { Meillet T. } 23 / 2 \text {, p. } 24-25\end{array}$ \\
\hline 1922 & & $\begin{array}{l}\text { Recueil des publications } \\
\text { scientifiques de Ferdinand de } \\
\text { Saussure } \\
\text { Cours de linguistique générale, } \\
2^{\mathrm{e}} \text { édition }\end{array}$ & $\begin{array}{l}\text { Meillet T. } 23 / 2 \text {, p. } 52 \\
\text { Meillet T. } 24 / 2 \text {, p. } 8\end{array}$ \\
\hline 1923 & $\begin{array}{l}\text { De Boer, Essai de syntaxe du français } \\
\text { moderne }\end{array}$ & & Meillet T. 24/2, p. 95-98 \\
\hline 1925 & Bally, Le langage et la vie, $2^{\mathrm{e}}$ édition & & Meillet T. 27/2, p. 14-16 \\
\hline 1926 & $\begin{array}{l}\text { Sechehaye, Essai sur la structure logique } \\
\text { de la phrase } \\
\text { Sechehaye, Abrégé de grammaire française } \\
\text { sur un plan constructif } \\
\text { De Boer, Essai sur la syntaxe moderne de la } \\
\text { préposition en français et en italien } \\
\text { Lips, Le Style indirect libre }\end{array}$ & & $\begin{array}{l}\text { Meillet T. } 27 / 2 \text {, p. } 1-2 \\
\text { Meillet T. } 28 / 2 \text {, p. } 159- \\
160 \\
\text { Meillet T. } 27 / 2 \text {, p. } 103- \\
105 \\
\text { Meillet T. } 28 / 2 \text {, p. } 46-48\end{array}$ \\
\hline 1927 & Karcevski, Système du verbe russe & $\begin{array}{l}\text { Sechehaye, « L'école genevoise } \\
\text { de linguistique générale » }\end{array}$ & Meillet T. 28/2, p. $42-44$ \\
\hline 1928 & & $\begin{array}{l}\text { Bally, Sechehaye, contribution } \\
\text { au } 1^{e r} \text { Congrès International des } \\
\text { Linguistes }\end{array}$ & Meillet T. 30/2, p. 24-25 \\
\hline 1929 & Frei, La grammaire des fautes & & $\begin{array}{l}\text { Meillet T. 30/2, p. } 145- \\
149\end{array}$ \\
\hline 1930 & Bally, La Crise du français & & Meillet T. 32/2, p. 123 \\
\hline 1931 & & $\begin{array}{l}2^{\mathrm{e}} \text { Congrès International de } \\
\text { Linguistes } \\
\text { Cours de linguistique générale, } \\
3^{\mathrm{e}} \text { édition }\end{array}$ & \\
\hline 1932 & $\begin{array}{l}\text { Bally, Linguistique générale et linguistique } \\
\text { française }\end{array}$ & & Meillet T. 34/2, p. 84-87 \\
\hline 1933 & $\begin{array}{l}\text { Journal de psychologie 30. Psychologie du } \\
\text { langage (articles de Bally et de Sechehaye) } \\
\text { De Boer, Introduction à l'étude de la } \\
\text { syntaxe du français }\end{array}$ & $\begin{array}{l}\text { Actes du } 2^{\mathrm{e}} \text { Congrès International } \\
\text { de Linguistes }\end{array}$ & $\begin{array}{l}\text { Meillet T. } 34 / 2 \text {, p. } 20-26 \\
\text { Bloch T. } 35 / 2 \text {, p. } 86-88 \\
\text { Meillet T. } 35 / 2 \text {, p. } 1\end{array}$ \\
\hline 1939 & $\begin{array}{l}\text { Adank, Essai sur les fondements } \\
\text { psychologiques de la métaphore affective }\end{array}$ & Mélanges Bally & $\begin{array}{l}\text { Cohen T. } 41 / 2 \text {, p. } 11-12 \\
\text { Cohen T. } 40 / 2 \text {, p. } 7-8\end{array}$ \\
\hline 1940 & & $\begin{array}{l}\text { Sechehaye, Bally, Frei " Pour } \\
\text { l'arbitraire du signe " } \\
\text { Création de la Société Genevoise } \\
\text { de Linguistique }\end{array}$ & \\
\hline
\end{tabular}

Fig. 1 : La production linguistique genevoise (1908-1940) 


\section{Les COMPTES-RENDUS DE LA SLP}

Parmi les auteurs des comptes-rendus des $B S L$ réunis dans la troisième colonne du tableau, le nom d'Antoine Meillet est récurrent; on ne s'en étonnera pas. «Âme de la SLP » (Boyer 1936, p. 196) et premier contributeur de la section, celui-ci se charge pendant la première guerre mondiale, de l'intégralité des comptes-rendus. Comme l'écrit Benveniste, « la Société a été si étroitement mêlée à son activité qu'on peut dire qu'il s'est identifié à elle pendant trente ans, de 1906 à 1936 » (1971, p. 27) La réception de la linguistique genevoise dans les $B S L$ est donc, en large partie, celle que lui a réservée Meillet.

Les comptes-rendus, on le remarque, portent plus sur les publications individuelles que sur les initiatives collectives. Cela tient à la rubrique considérée dont la fonction est de réagir au coup par coup à des publications isolées. Les comptes-rendus construisent donc un point de vue fragmentaire et discontinu sur la production linguistique genevoise, percevant d'abord la pluralité là où d'autres sections - celle des articles ou des séances - auraient peut-être mis davantage en lumière l'unité. Cependant les initiatives concentriques d'école, même si elles ne sont pas toutes prises en charge par les comptes-rendus, influent sur la réception des publications individuelles. La perception diffuse d'une unité transparaît dans les recensions par la récurrence des renvois à d'autres linguistes genevois. Les comptes-rendus mettent rarement en relation les travaux des linguistes sur un plan horizontal - un seul renvoi aux travaux de Sechehaye dans les comptes-rendus des livres de Bally par exemple (BSL 27/2, p. 45) -, mais toujours verticalement, en termes de filiation. Ainsi Meillet ne manque jamais de rappeler que Bally, Sechehaye, Gautier ou Regard sont les élèves de Saussure, ou qu'Henri Frei et Marguerite Lips sont les élèves de Bally. Quant à la reconnaissance d'une " école genevoise », elle apparaît explicite dans les comptes-rendus des $B S L$ à trois reprises : en 1927, où Meillet parle d'un « groupe de linguistes genevois » incluant Bally et Sechehaye (BSL 27/2, p. 45); en 1928, au sujet de Marguerite Lips qui, écrit Meillet, « se réclame de l'école genevoise » (BSL 28/2, p. 46) ; et en 1931 au sujet de la première livraison des Travaux du Cercle Linguistique de Prague. Cette dernière recension est révélatrice des conflits déterminatifs auxquels donne alors lieu le syntagme « école genevoise ». Très positif, si l'on excepte la critique du « pédantisme » des thèses du Cercle, que Meillet trouve « rébarbatives », le compte-rendu se clôt sur ce constat : «Les idées dont s'inspire le groupe procèdent de l'école genevoise qui est sortie de l'enseignement de F. de Saussure, tel qu'il est connu par les cours publiés après la mort du maître » (Meillet 1931, p. 18). Troubetzkoy déplore, dans sa correspondance, qu'on assimile le CLP « purement et simplement à l'École de Saussure ", d'autant plus qu'il critique sévèrement « la direction qu'a prise 
l'activité de ses élèves » (2006, p. 287 et 348). De fait, sous la plume de Meillet, « école genevoise » ne désigne ni les linguistes de Genève ni leurs travaux, ou alors seulement leur travail éditorial autour de l'enseignement de Saussure. En fait, «école genevoise » équivaut ici à Cours de linguistique générale.

C'est dans le compte-rendu des Actes du $2^{e}$ congrès International de Linguistes que Meillet reconnaît pour la seule fois explicitement une spécificité de la linguistique développée à Genève :

Le premier congrès de linguistes, réuni par les soins de M. Schrijnen, s'était montré si utile que les linguistes ont répondu avec empressement à l'invitation des linguistes genevois, disciples de F. de Saussure. Le congrès préparé par eux avait dès l'abord un caractère neuf et original. En un sens il continuait le premier dont le trait frappant avait été que la grammaire comparée des langues indoeuropéennes, qui tient presque partout une place dominante dans les études de linguistique historique, n'y avait pas été au premier plan. À Genève, en grande partie sous l'influence des organisateurs, c'est sur la grammaire générale qu'ont porté les exposés les plus nombreux et souvent les plus marquants. L'exposé du prince Troubetzkoy sur la phonologie a produit une forte impression. On est heureux de voir avec quelle conscience et quelle habileté les linguistes genevois ont donné un aperçu exact d'un congrès qui a été remarquablement riche et varié. Ceux qui ont participé à ce congrès y retrouvent leurs impressions. (BSL $35 / 2$, p. 1)

La spécificité reconnue aux disciples genevois de Saussure est d'accélérer l'essor, amorcé à La Haye, d'une linguistique générale. Surtout, Meillet conçoit dans le champ linguistique une relation à deux termes : la grammaire comparée - et, ici, la grammaire générale utilisée en lieu et place du syntagme " linguistique générale » promu dans le congrès, sans que l'on puisse identifier si la substitution est indifférente ou si elle contient une critique voilée ${ }^{5}$. Or le discours inaugural de Bally, retranscrit dans les actes du congrès dont Meillet rend compte, distingue trois tendances dans la linguistique de son temps : (1) l'enquête sur les langues du monde, portée au congrès de La Haye par Antoine Meillet, (2) la description phonologique des langues, (3) «le souffle de spiritualité [qui] anime aujourd'hui la linguistique » et que l'école de Genève entend incarner (Bally 1933, p. 30). De ces trois tendances - Paris, Prague, Genève si on veut les localiser -, Meillet n'en retient que deux et cite, pour le courant général, la phonologie de Troubetzkoy et non les efforts des genevois pour faire de la linguistique « une science de l'esprit».

5 En faveur de la seconde hypothèse on peut invoquer un texte contemporain où Meillet écrit au sujet de l'Essai de Sémantique de Bréal et de sa réhabilitation de la grammaire générale des Idéologues : « le livre pouvait paraître réactionnaire en 1897 ; aujourd'hui certaines des tendances actuelles le rejoignent, en partie avec moins de justesse et de précision. Si le livre avait eu en son temps un beau succès, il semble que, maintenant, on ne le lise pas autant qu'il faudrait. Le rationalisme de Bréal déplaît sans doute à des novateurs qui mettent au premier plan les faits affectifs » (Meillet 1932, p. 289). Difficile de ne pas voir dans cette dernière phrase une allusion à Bally. 
Concernant, enfin, les publications individuelles, il est possible de dégager, par-delà les remarques induites par la spécificité de chaque ouvrage, quelques traits constants de la réception des travaux genevois. Aucune de ces publications ne relève de la linguistique historique, même les livres de Regard sur la koinè sont reçus comme un travail de «linguistique synchronique » (BSL 21, p. 213). Surtout, toutes ou presque portent sur le français contemporain. Or, comme l'a remarqué Claire Blanche-Benveniste dans une étude sur la place de la linguistique du français dans les $B S L$ (2005, p. 187-207), les comptes-rendus de Meillet sur la langue française sont presque systématiquement négatifs ${ }^{6}$. La production genevoise ne fait pas exception à cette sévérité. Seul Bally bénéficie d'une relative clémence : son Traité de stylistique française est bien reçu, « il inaugure », selon Meillet, « un ordre de recherches nouvelles dont on doit attendre un rajeunissement scientifique » (BSL 16, p. 118). L'accueil réservé aux ouvrages suivants est plus mesuré mais fait toujours état de l'originalité de l'approche de Bally. Les travaux de Sechehaye, en revanche, ne suscitent guère l'intérêt : ses ouvrages de 1908 ne sont pas pris en compte dans les $B S L$, et ceux de 1926 ne font l'objet que de recensions expéditives. D'une manière générale, Meillet pointe chez les genevois des problèmes de méthode dans la description du français contemporain. La nécessité de « délimiter l'objet étudié » (BSL 24/2, p. 95), c'est-à-dire de hiérarchiser et de déterminer géographiquement et socialement les données, fonde une partie des critiques adressées à Linguistique générale et linguistique française de Bally, à $L a$ Grammaire des fautes de Frei et aux travaux de syntaxe de De Boer. Cette critique n'est toutefois pas réservée aux genevois : elle est le défaut majeur que Meillet dénonce dans les travaux de son temps sur la langue française. Il y a cependant aussi des constantes dans les comptes-rendus qui semblent spécifiques à la réception de la linguistique genevoise.

La première est le rappel récurrent d'une filiation saussurienne, mais dont l'apport conceptuel se réduit toujours à la seule distinction diachronie/synchronie. La mention de ce principe partagé s'accompagne parfois de la critique, vis-à-vis de la description linguistique pratiquée par les genevois, d'un synchronisme intransigeant qui ne tiendrait pas compte des apports de la linguistique historique. Ainsi Meillet objecte à Bally qu' « il n'y a pas besoin d'ignorer l'histoire de la langue

6 Cette sévérité est à mettre en relation avec le projet d'ouvrage sur la langue française auquel Meillet travaille à la fin des années 1910 et au début des années 1930 : «Dans les dernières années de sa vie, il [Meillet] avait souvent entretenu ses amis d'un projet d'ouvrage sur le développement de la langue française. Cet ouvrage aurait formé une sorte de triptyque avec ceux qu'il avait écrits sur le grec et le latin. Il en aurait été le panneau central. Meillet devait poursuivre l'histoire de notre langue jusqu'à l'époque contemporaine. C'est un regret cuisant que le temps lui ait manqué pour produire une pareille œuvre, qui aurait été si riche d'enseignements sur le passé et sur le présent de notre langue » (Vendryes 1937, p. 27). Les esquisses manuscrites de ces projets ont été retrouvées et publiées par Granucci en 1992. 
pour rendre un compte exact de son présent, et peut-être au contraire » (BSL 18, p. 181). Le même reproche est adressé à La Grammaire des fautes : «M. Frei est trop soucieux de réagir contre les procédés de la linguistique historique pour analyser avec précision les faits dont il tire parti » (BSL 30/2, p. 148).

Un autre trait spécifique de la réception de la production genevoise est la critique systématique de développements caractérisés négativement comme « philosophiques », « logiques » ou « abstraits ». Meillet écrit ainsi que Bally, « a trop développé des principes de philosophie courante moins originaux que ses idées sur la linguistique » $(B S L 18$, p. 180) ; Sechehaye se voit reprocher des « digressions philosophiques inutiles au grammairien » (BSL 27/2, p. 1), Karcevski un « vocabulaire logique et mathématique souvent rebutant» (BSL 28/2, p. 42), Frei des « phrases vagues pleines de termes abstraits » (BSL 30/2, p. 149), De Boer des « divisions d'ordre psychique (ou logique), [et] non d'ordre linguistique » (BSL 27/2, p. 104). La critique de l'abstraction trouve des traductions précises dans l'analyse des descriptions linguistiques proposées. Meillet fait ainsi part, à plusieurs reprises, de son désaccord avec une approche linguistique qui fonde, en principe, l'activité psychologique et non l'expression linguistique :

M. Bally croit que, pour aboutir à étudier le langage en tant que moyen d'action et moyen d'expression, il faudrait partir « de la pensée et de la vie »; on ne pourra se rendre compte des résultats qu'on obtiendrait ainsi qu'une fois que l'étude sera faite. Il est à craindre qu'elle ne soit bien difficile et que les conclusions ne manquent de précision. La conférence de $\mathrm{M}$. Bally n'exprime à ce point de vue qu'un désir, elle ne fournit même pas à proprement parler un programme de recherches. (BSL 18, p. 181)

Entre un linguiste qui pense comme M. C. de Boer et un linguiste qui pense comme moi, il y a un malentendu initial. « Pour les linguistes, écrit M. de Boer, pour qui, dans les langues, les fonctions disparaissent avec les formes tout ce chapitre sera chimérique » (p. 35).

Mais je crois que ce malentendu est plutôt verbal que réel. La linguistique n'a pas à faire une théorie de la réalité ni même de la manière dont l'homme conçoit la réalité. Elle n'a à considérer que l'expression que reçoivent dans les langues, les réalités et les conceptions. Que l'on parte de l'expression linguistique pour en examiner le sens ou du sens pour en examiner l'expression linguistique, c'est affaire de procédé. On peut, suivant le cas, avoir avantage à opérer d'une manière ou de l'autre ; cela ne change rien au fait que l'unique objet de la linguistique est l'expression donnée par la langue aux conceptions. (BSL 27/2, p. 103)

Ni l'angle de l'expressivité, cher à Bally, ni celui des structures logiques sousjacentes à la langue recherché par Sechehaye et De Boer ne trouvent grâce à ses yeux. En conséquence, il récuse aussi bien l'explication par l'ellipse proposée par Bally pour les phrases exclamatives comme Le pauvre homme! (BSL 27/2, p. 45) que les distinctions opérées par De Boer dans le système prépositionnel du français 
qui n'ont pas de correspondance morphologique (BSL 27/2, p. 103-105). Partir des faits linguistiques et non de la pensée, du signe et non du sens : c'est la leçon qu'administre Meillet à plusieurs reprises dans ses comptes-rendus.

En définitive, «l'école linguistique de Genève » est, du point de vue de la SLP, une expression au référent passablement indéterminé. Elle apparaît dans les $B S L$ à la fin des années 1920 dans le sillage du premier Congrès international de La Haye pour désigner une nébuleuse, incluant aussi bien les genevois que les praguois, réunie autour d'un principal critère : citer ou discuter le Cours de linguistique générale. Pour le reste, si, à Paris, le groupe social des linguistes de Genève est bien identifié, aucune unité théorique ne lui est attribuée d'emblée. Le recoupement des comptes-rendus de leurs différents travaux fait ressortir les deux caractéristiques qui leur sont attachées : l'approche synchronique d'une part, l'appréhension de la langue comme « opération de l'esprit » (Bally 1933, p. 30) d'autre part, ce que Meillet assimile à de la " grammaire générale ». En critiquant le manque d'immanence de leur approche linguistique, Meillet entrevoit le saussurisme paradoxal des deux éditeurs du $C L G$, qu'a mis en évidence l'historiographie', et qui est largement tributaire de contacts avec la linguistique et la psychologie allemandes, indépendants de l'enseignement saussurien.

\section{BiBLIOGRAPHIE}

\section{Sources primaires}

Les comptes-rendus des BSL qui figurent dans la Fig. 1 ne sont pas repris dans la Bibliographie.

Bally, Charles, 1933. «Discours d'ouverture », Actes du deuxième congrès international de linguistes, Genève, 25-29 août 1931, Paris, Maisonneuve, 29-30.

Bally, Charles et Sechehaye, Albert, 1930. «Quelles sont les méthodes les mieux appropriées à un exposé complet et pratique de la grammaire d'une langue quelconque? », Actes $d u$ premier Congrès international de linguistes à La Haye : Du 10-15 avril 1928, Leyde, Sithoff, 36-53.

Benveniste, Émile, 1971. "Allocution de la célébration du centenaire de la SLP », BSL 66/1, 19-34.

Boyer, Paul, 1936. "Antoine Meillet. L'homme et le savant », Revue des études slaves 16, 191-198.

Cahen, Maurice, 1925. «Festskrift tillägnad Hugo Pipping på hans sextioårsdag den 5 november $1924 », B S L$ 26/2, 162-173.

Cohen, Marcel, 1945. « Cahiers Ferdinand de Saussure 1 et 2 », BSL 42/2, 1-2.

Ernout, Alfred, 1922. « J. Vendryes. Le langage. Introduction linguistique à l'histoire ", Journal des savants 20/3, 134-136.

Gauthiot, Robert, 1914. "Ferdinand de Saussure (1857-1913). Notice », Bulletin de l'Association des élèves et des anciens élèves de l'École Pratique des Haute Études (Section des Sciences historiques et philologiques), 49-55.

Grammont, Maurice, 1912. «Mélanges linguistiques offerts à M. F. de Saussure. Compterendu », Revue des Langues Romanes 55, 387-389. 
Meillet, Antoine, [1906] 1921. "L'état actuel des études de linguistique générale », Linguistique historique et linguistique générale I, Paris, Hachette.

- 1920. « Hirt H. Des indogermanische Vokalismus », BSL 22, 190-192.

- 1926-1927. «Stolz-Schmalz, Lateinische Grammatik», BSL 27/2, 61-70.

- 1928, « Avant-Propos », Étrennes de linguistique offertes par quelques amis à Émile Benveniste, Paris, Geuthner, 5-7.

- 1931, " Travaux du cercle linguistique de Prague, 1929 », BSL 31/2, 18-20.

- 1932. « La grammaire comparée au Collège de France », Lefranc Abel et alii (éds), Le Collège de France (1530-1930), Paris, PUF, 279-289.

Meillet, Antoine et Bally, Charles, [1908] 2006. «Allocutions prononcées le 14 juillet 1908 à l'occasion de la remise à Ferdinand de Saussure des mélanges de linguistique » éd. René Amacker, Cahiers Ferdinand de Saussure 59, 179-185.

Sechehaye, Albert, 1927. " L'école genevoise de linguistique générale », Indogermanische Forschungen 44, 217-240.

- [1939] 2001. " Allocutions prononcée le $1^{\text {er }}$ juillet 1939 à l'occasion de la remise à Charles Bally des Mélanges linguistiques » éd. Anne-Marguerite Fryba-Reber, Cahiers Ferdinand de Saussure 54, 559-462.

- «Les sociétés savantes. La Société Genevoise de Linguistique », Revue universitaire suisse 15/3, 175-177.

Sechehaye, Albert, Bally, Charles et Frei, Henri, 1940-1941. « Pour l'arbitraire du signe », Acta linguistica 2, 165-169.

Sommerfelt, Alf, 1924. « La philosophie linguistique française. Réponse à M. Hjalmar Falk », BSL 25/1, 22-33.

- [1932] 1966. " La linguistique, science sociologique », Diachronic and Synchronic Aspects of Language : Selected articles, La Haye, Mouton, 36-51.

Troubetzkoy Nicolaï, 2006. Correspondance avec Roman Jakobson et autres écrits, éd. Patrick Sériot, Payot, Lausanne.

Vendryes, Joseph, 1937. «Antoine Meillet », BSL 38/1, 1-42.

- 1955. « Première société linguistique. La Société de linguistique de Paris (1865-1955)», Orbis 4, 7-21.

\section{Sources secondaires}

Amacker, René, 2000. "Le développement des idées saussuriennes chez Bally et Sechehaye », Historiographia Linguistica 27/2, 205-264.

Amacker, René, Forel, Claire, Fryba-Reber, Anne-Marguerite, 1997. " Les Cahiers Ferdinand de Saussure des origines à nos jours ", Cahiers Ferdinand de Saussure 50, 341-360.

Amsterdamska, Olga, 1987. School of Thought : the development of linguistics from Bopp to Saussure, La Haye, Mouton.

Bergounioux, Gabriel, 1997. « La Société linguistique de Paris (1876-1914) », BSL 92/1, $1-26$.

- 2005. « La Société de linguistique de Paris dans son histoire », BSL 100/1, 359-390.

Blanche-Benveniste, Claire, 2005. « La place de la langue française dans les Mémoires et le Bulletin de la Société de linguistique de Paris », BSL 100/1, 183-223.

Chevalier, Jean-Claude, 1997. « Troubetzkoy, Jakobson et la France, 1919-1939 », Cahiers de l'ILSL 9, 31-43.

- 1999. "Albert Sechehaye, pédagogue et théoricien », Cahiers Ferdinand de Saussure $52,89-81$.

- 2000. « Les congrès internationaux et la linguistique », Auroux, Sylvain (éd.), Histoire des idées linguistiques 3, Liège-Paris, Mardaga, 517-528. 
Fryba-Reber, Anne-Marguerite, 1994. Albert Sechehaye et la syntaxe imaginative, Droz, Genève.

- 2001. «De la cohésion et de la fluidité de la langue. Textes inédits (1908-1943) de Charles Bally et d'Albert Sechehaye », Cahiers Ferdinand de Saussure 54, 429-487.

- 2013. Philologie et linguistique romanes. Institutionnalisation des disciplines dans les universités suisses (1872-1945), Leuven - Paris, Peeters.

Granucci, Fiorenza, 1992. "Complementi a "Pour un manuel de linguistique générale" di Antoine Meillet », Studi e saggi linguistici 32, 13-232.

Joseph, John E., 2012. Saussure, Oxford, Oxford University Press.

Lamberterie, Charles de, 2005. « La grammaire comparée des langues indo-européennes dans les MSL et le BSL des origines au règne d'Antoine Meillet », BSL 100/1, 17-44.

Redard, Georges, 1982. "Charles Bally disciple de Ferdinand de Saussure », Cahiers Ferdinand de Saussure 36, 3-23.

Savatovsky, Dan, 2004. « Comment faire école ? (Saussure à Paris, II)», Cahiers Ferdinand de Saussure 56, 311-326.

Segre, Cesare, 1985. «In memoriam André Burger », Cahiers Ferdinand de Saussure 39, 3-8.

Servos, John W., 1993. « Research Schools and their histories », Osiris 8, 3-15.

Waquet, Françoise, 2008. Les enfants de Socrate. Filiation intellectuelle et transmission du savoir. XVII ${ }^{e}-X X I^{e}$ siècle, Paris, Albin Michel. 